\title{
Neutrino oscillations and instabilities in degenerate relativistic astrophysical plasmas
}

\author{
Fernando Haas* \\ Instituto de Física, Universidade Federal do Rio Grande do Sul, Av. Bento Gonçalves 9500, 91501-970 Porto Alegre, RS, Brasil
}

(Received 7 May 2019; revised manuscript received 13 June 2019; published 27 June 2019)

\begin{abstract}
We set up a proposal to extend significantly recent works on neutrino-plasma interaction, allowing the possibility of deep degenerate and relativistic electrons, which are often present in compact stars such as high-density white dwarfs. The methodology involves the covariant hydrodynamic formulation of ultradense plasmas. We propose the generalization of previous studies, on the interaction between ion-acoustic waves and resonant neutrino flavor oscillations in a mixed neutrino beam, admitting degenerate and relativistic electron populations. Destabilization of the ion acoustic wave has higher growth rates, thanks to the very high densities present in plasmas under these extreme conditions. We take into account applications to white dwarf stars in the process of collapsing, producing type II supernovas.
\end{abstract}

DOI: 10.1103/PhysRevE.99.063209

\section{INTRODUCTION}

Neutrinos play a decisive role in a variety of fields, such as cosmology, particle physics, and astrophysics. The 2015 Nobel Prize [1], in particular, was awarded to T. Kajita and A. B. McDonald for the experimental confirmation of neutrino flavor oscillations, showing the existence of a neutrino mass and pointing out the incompleteness of the standard model.

In the process of gravitational collapse, the extreme densities obtained in the core enhance the inverse beta decay rates, resulting in strong neutrino winds propagating out from the protoneutron star. Neutrino beams are believed to be responsible for plasma heating in the stalled shock in type II supernova explosions [2-4]. In this context, for the supernova SN1987A, a burst of $10^{58}$ neutrinos of all flavors radiating in a few seconds was observed [5]. Sources of anisotropic neutrino beams have also been investigated [6]. Moreover, neutrinos play a central role in the lepton era of the early universe [7].

Recently, a model for the neutrino-plasma interaction taking into account neutrino oscillations was proposed [8], assuming for simplicity nondegenerate and nonrelativistic electrons. However, in compact stars these restrictions are violated easily, with strongly degenerate and relativistic electrons due to Fermi velocities near the speed of light. The purpose of the present work [9] is to improve significantly the previous treatment, in terms of the hydrodynamic model arising from the perfect relativistic fluid equations [10] modified by the neutrino force. Our aim is to consider the destabilization of ion-acoustic waves driven by neutrino beams, evaluating the associate instability growth rates in extreme scenarios. Previously, neutrino oscillations in nonrelativistic plasmas have also been analyzed in [11] and [12], taking into account the collisional damping of ion-acoustic waves. In addition, neutrino-magnetohydrodynamic modes $[13,14]$ and neutrinomodified wave propagation in strongly magnetized plasma
[15] have been considered, without the inclusion of flavor oscillations, degeneracy, or relativistic effects.

The article is organized as follows. In Sec. II, the basic fluid model for degenerate relativistic electrons and cold nonrelativistic ions is written, coupled to a two-flavor neutrino beam taking into account neutrino oscillations. In Sec. III, the linear dispersion relation for ion-acoustic waves is derived. Assuming a double-resonance condition between the ion-acoustic wave, the neutrino beam, and the neutrino oscillations, the appropriate instability growth rate is obtained. In Sec. IV, the growth rate is evaluated for parameters compatible with type II supernovas. The time scale of the instability and the unstable wavelengths are calculated, allowing us to estimate the impact of neutrino oscillations. Section V is reserved for the conclusions.

\section{BASIC MODEL}

For simplicity, we consider a two-flavor neutrino model. The model is given by a hydrodynamical model for electrons, ions, electron-neutrinos, and muon-neutrinos. Denoting $n_{e, i}$ and $\mathbf{u}_{e, i}$ as, respectively, the electron (e) and ion (i) proper fluid densities and velocity fields, one has the relativistic electron continuity and force equations,

$$
\begin{aligned}
& \frac{\partial\left(\gamma n_{e}\right)}{\partial t}+\nabla \cdot\left(\gamma n_{e} \mathbf{u}_{e}\right)=0, \quad \gamma=\left(1-\left|\mathbf{u}_{e}\right|^{2} / c^{2}\right)^{-1 / 2}, \\
& m_{e} H\left(\frac{\partial}{\partial t}+\mathbf{u}_{e} \cdot \nabla\right)\left(\gamma \mathbf{u}_{e}\right) \\
& =-\frac{\gamma}{n_{e}}\left(\nabla+\frac{\mathbf{u}_{e}}{c^{2}} \frac{\partial}{\partial t}\right) P+e \nabla \phi+\sqrt{2} G_{F}\left(\mathbf{E}_{v}+\mathbf{u}_{e} \times \mathbf{B}_{v}\right),
\end{aligned}
$$

together with the corresponding nonrelativistic equations for cold ions,

$$
\frac{\partial n_{i}}{\partial t}+\nabla \cdot\left(n_{i} \mathbf{u}_{i}\right)=0, \quad m_{i}\left(\frac{\partial}{\partial t}+\mathbf{u}_{i} \cdot \nabla\right) \mathbf{u}_{i}=-Z e \nabla \phi .
$$


In Eqs. (1)-(3), $m_{e, i}$ are the electron (charge $-e$ ) and ion (charge $Z e$ ) masses, $c$ is the speed of light, $G_{F}$ is the Fermi coupling constant, and $\phi$ is the electrostatic potential. In addition, $\mathbf{E}_{v}$ and $\mathbf{B}_{v}$ are the effective neutrino electric and magnetic fields defined by

$$
\mathbf{E}_{v}=-\nabla N_{e}-\frac{1}{c^{2}} \frac{\partial}{\partial t}\left(N_{e} \mathbf{v}_{e}\right), \quad \mathbf{B}_{v}=\frac{1}{c^{2}} \nabla \times\left(N_{e} \mathbf{v}_{e}\right),
$$

in terms of the electron-neutrino fluid density and velocity field $N_{e}$ and $\mathbf{v}_{e}$. We also have Poisson's equation,

$$
\nabla^{2} \phi=\frac{e}{\varepsilon_{0}}\left(\gamma n_{e}-Z n_{i}\right),
$$

where $\varepsilon_{0}$ is the vacuum permittivity constant. The Fermi weak force couples electrons (leptons) to electron-neutrinos. Finally, to determine the pressure $P=P\left(n_{e}\right)$ for a fully degenerate electron gas we use Chandrasekhar's [16] barotropic equation of state,

$$
\begin{gathered}
\frac{P}{n_{0} m_{e} c^{2}}=\frac{1}{8 \zeta_{0}^{3}}\left[\zeta\left(2 \zeta^{2}-3\right) \sqrt{1+\zeta^{2}}+3 \sinh ^{-1} \zeta\right], \\
\zeta=\frac{\hbar}{m_{e} c}\left(3 \pi^{2} n_{e}\right)^{1 / 3}=\zeta_{0}\left(\frac{n_{e}}{n_{0}}\right)^{1 / 3}, \quad \zeta_{0}=\frac{p_{F}}{m_{e} c},
\end{gathered}
$$

where $n_{0}$ is the equilibrium electron number density, $\hbar$ is the reduced Planck constant, and $p_{F}=\hbar\left(3 \pi^{2} n_{0}\right)^{1 / 3}$ is the electron Fermi momentum, yielding the specific enthalpy $H=\int d P /\left(m_{e} c^{2} n_{e}\right)=\sqrt{1+\zeta^{2}}$, responsible for the relativistic electron mass increase due to a high Fermi velocity. It is important to note that the Chandrasekhar equation of state gives a linear dispersion for ion-acoustic waves in the absence of neutrinos, which agrees with the result from the relativistic Vlasov equation, in the long-wavelength limit [17]. In addition, magnetized plasmas can be treated by simply including the magnetic force on electrons and ions [15]. In the case of electromagnetic waves, also the full set of Maxwell equations would be necessary $[13,14]$.

To close the system, one needs the equations for the twoflavor oscillations. One has $[8,12]$

$$
\begin{gathered}
\frac{\partial N_{e}}{\partial t}+\nabla \cdot\left(N_{e} \mathbf{v}_{e}\right)=\frac{1}{2} N \Omega_{0} P_{2}, \\
\frac{\partial N_{\mu}}{\partial t}+\nabla \cdot\left(N_{\mu} \mathbf{v}_{\mu}\right)=-\frac{1}{2} N \Omega_{0} P_{2},
\end{gathered}
$$

where $N_{\mu}$ and $\mathbf{v}_{\mu}$ are the muon-neutrino fluid density and velocity field, $N=N_{e}+N_{\mu}$ is the total neutrino fluid density, and $P_{2}$ represents the quantum coherence in the flavor polarization vector $\mathbf{P}=\left(P_{1}, P_{2}, P_{3}\right)$. In addition, $\Omega_{0}=\omega_{0} \sin 2 \theta_{0}$, where $\omega_{0}=\Delta m^{2} c^{4} /\left(2 \hbar \mathcal{E}_{0}\right)$ with a squared neutrino mass difference $\Delta m^{2}$. Finally, $\mathcal{E}_{0}$ is the neutrino spinor's energy in the fundamental state and $\theta_{0}$ is the neutrino oscillation mixing angle. Note the conservation law

$$
\frac{d}{d t} \int\left(N_{e}+N_{\mu}\right) d^{3} r=0,
$$

following, for instance, from decaying or periodic boundary conditions.
Representing the (ultra)relativistic electron- and muonneutrino momenta by $\mathbf{p}_{e}=\mathcal{E}_{e} \mathbf{v}_{e} / c^{2}$ and $\mathbf{p}_{\mu}=\mathcal{E}_{\mu} \mathbf{v}_{\mu} / c^{2}$, where $\mathcal{E}_{e}$ and $\mathcal{E}_{\mu}$ are the corresponding neutrino beam energies, the neutrino force equations read

$$
\begin{aligned}
& \frac{\partial \mathbf{p}_{e}}{\partial t}+\mathbf{v}_{e} \cdot \nabla \mathbf{p}_{e}= \sqrt{2} G_{F}\left(-\nabla\left(\gamma n_{e}\right)-\frac{1}{c^{2}} \frac{\partial}{\partial t}\left(\gamma n_{e} \mathbf{u}_{e}\right)\right. \\
&\left.+\frac{\mathbf{v}_{e}}{c^{2}} \times\left[\nabla \times\left(\gamma n_{e} \mathbf{u}_{e}\right)\right]\right) \\
& \frac{\partial \mathbf{p}_{\mu}}{\partial t}+\mathbf{v}_{\mu} \cdot \nabla \mathbf{p}_{\mu}=0
\end{aligned}
$$

The neutrino-plasma interaction model was derived from an action principle [18,19], in the absence of flavor oscillations and for nonrelativistic electrons; see also [20] for the treatment of neutrino-modified Langmuir waves.

To conclude, the flavor polarization vector $\mathbf{P}=\left(P_{1}, P_{2}, P_{3}\right)$ evolves in a material medium according to

$$
\begin{aligned}
\frac{\partial P_{1}}{\partial t} & =-\Omega\left(n_{e}\right) P_{2}, \quad \frac{\partial P_{2}}{\partial t}=\Omega\left(n_{e}\right) P_{1}-\Omega_{0} P_{3}, \\
\frac{\partial P_{3}}{\partial t} & =\Omega_{0} P_{2},
\end{aligned}
$$

where $\Omega\left(n_{e}\right)=\omega_{0}\left[\cos 2 \theta_{0}-\sqrt{2} G_{F} n_{e} /\left(\hbar \omega_{0}\right)\right]$. Equation (12) was derived [21,22] for neutrinos traveling in a fixed, static background, so that $\Omega\left(n_{e}\right)$ involves the proper electron density $n_{e}$.

Overall, there are 20 equations defined by Eqs. (1)-(3), (5), (8), and (10)-(12), for 20 variables, namely, the quantities $n_{e, i}, \mathbf{u}_{e, i}, \phi, N_{e, \mu}, \mathbf{v}_{e, \mu}$, and $P_{1,2,3}$. We observe that the electron- and muon-neutrino energies $\mathcal{E}_{e, \mu}$ are functions of the corresponding momenta by means of the usual relativistic energy-momentum relation $\mathcal{E}_{v}=\left(p_{v}^{2} c^{2}+m_{v}^{2} c^{4}\right)^{1 / 2}, v=e, \mu$. A neutrino mass $m_{v}$ is assumed just for the sake of the calculation (at the end it disappears). Specifically, the procedure is detailed in Appendix A of Ref. [8].

\section{LINEAR WAVES}

The system (1)-(3), (5), (8), and (10)-(12) admits the equilibrium

$$
\begin{aligned}
& n_{e}=n_{0}, \quad n_{i}=n_{0} / Z, \quad \mathbf{u}_{e}=\mathbf{u}_{i}=0, \quad \phi=0 \\
& N_{e}=N_{e 0}, \quad N_{\mu}=N_{\mu 0}, \quad \mathbf{v}_{e}=\mathbf{v}_{\mu}=\mathbf{v}_{0}, \\
& P_{1}=\frac{\Omega_{0}}{\Omega_{v}}, \quad P_{2}=0, \quad P_{3}=\frac{\Omega\left(n_{0}\right)}{\Omega_{v}}=\frac{N_{e 0}-N_{\mu 0}}{N_{0}},
\end{aligned}
$$

where $\Omega_{v}=\sqrt{\Omega^{2}\left(n_{0}\right)+\Omega_{0}^{2}}$ is the neutrino-flavor oscillation frequency. Linearizing, taking plane-wave perturbations $\sim \exp [i(\mathbf{k} \cdot \mathbf{r}-\omega t)]$, and following the same procedure detailed in [8], the result is

$$
\begin{aligned}
\omega^{2}= & c_{s}^{2} k^{2}+\frac{\Delta_{e} c^{2} k^{2} \Lambda(\theta)\left(c^{2} k^{2}-\omega^{2}\right)}{\left(\omega-\mathbf{k} \cdot \mathbf{v}_{0}\right)^{2}} \\
& +\frac{\Delta \Omega_{0}^{2} \omega \mathcal{E}_{0}\left(c^{2} k^{2}-\omega \mathbf{k} \cdot \mathbf{v}_{0}\right)}{2 \hbar \Omega_{v}\left(\omega-\mathbf{k} \cdot \mathbf{v}_{0}\right)\left(\omega^{2}-\Omega_{v}^{2}\right)}
\end{aligned}
$$


where

$$
\begin{aligned}
\Delta_{e} & =\frac{2 G_{F}^{2} N_{e 0} n_{0}}{m_{i} c^{2} \mathcal{E}_{0}}, \quad \Delta=\frac{2 G_{F}^{2} N_{0} n_{0}}{m_{i} c^{2} \mathcal{E}_{0}}, \\
\Lambda(\theta) & =\left(1-\frac{v_{0}^{2}}{c^{2}}\right) \cos ^{2} \theta+\sin ^{2} \theta,
\end{aligned}
$$

with $\mathbf{k} \cdot \mathbf{v}_{0}=k v_{0} \cos \theta$ and where the ion-acoustic speed $c_{s}$ in fully degenerate relativistic plasmas [17] is given by

$$
c_{s}^{2}=\frac{Z p_{F}^{2}}{3 m_{e} m_{i} \sqrt{1+\xi_{0}^{2}}},
$$

assuming completely ionized plasma with ionic atomic number $Z$. In comparison with [8], the only difference is the improved ion-acoustic speed, now adapted to fully degenerate and relativistic plasma and, additionally, allowing for $Z \neq 1$. It was assumed that $\omega \ll \omega_{\text {pi }}=\sqrt{Z n_{0} e^{2} /\left(m_{i} \varepsilon_{0}\right)}$ and $\mathcal{E}_{0}=\mathcal{E}_{e 0} \approx \mathcal{E}_{\mu 0}$. By inspection, it is not surprising that the dispersion relation, (14), is formally identical to the result in [8] (with a new expression for the ion-acoustic speed), since in the linearization procedure one has $\gamma \approx 1$ due to the absence of relativistic streaming electrons.

Besides the traditional ion-acoustic mode, Eq. (14) shows two contributions, one proportional to $\Delta_{e}$, which is due to the energy seed by the streaming neutrinos, while the term proportional to $\Delta$ is due to coupling with the neutrino oscillations. In the absence of a squared neutrino mass difference $\left(\Delta m^{2}=0\right)$ one has $\Omega_{0}^{2}=0$ so that the last term in Eq. (14) disappears. If we formally set $\Delta=0$, one regains the result in [23], with a modified ion-acoustic speed, taking into account that $c_{s} \ll c$ except for huge densities typical of neutron stars, outside the scope of the model.

\section{INSTABILITY OF THE ION-ACOUSTIC WAVE}

In view of the small value $G_{F}=4.62 \times 10^{-62} \mathrm{~J} \cdot \mathrm{m}^{3}$ of the Fermi constant, the neutrino contribution in Eq. (14) will typically be a small perturbation. The neutrino effect on ion-acoustic waves without neutrino oscillations has been essentially examined in [23]. Therefore, it is more convenient to focus on the case where neutrino oscillations can have a significant influence. Hence we assume the double-resonance condition

$$
\omega \approx c_{s} k=\Omega_{v}=\mathbf{k} \cdot \mathbf{v}_{0},
$$

enhancing the last term on the right-hand side of the dispersion relation, (14). Physically, Eq. (17) shows the (almost) resonance of the ion-acoustic wave with the streaming neutrinos and with the flavor oscillations, which carry an energy which can be exchanged with the wave.

Assuming Eq. (17) we set

$$
\omega=\Omega_{v}+\delta \omega, \quad|\delta \omega| \ll \Omega_{v}
$$

in Eq. (14). Taking into account ultrarelativistic neutrinos $\left(v_{0} \approx c\right)$, one gets $\cos \theta=c_{s} / c \ll 1$ (a filamentationlike instability), $\omega \ll c k$, so that $\Lambda(\theta) \approx 1$ and

$$
(\delta \omega)^{3}=\frac{\Delta_{e}}{2}\left(\frac{c}{c_{s}}\right)^{4} \Omega_{v}^{3}+\frac{\Delta \Omega_{0}^{2} \mathcal{E}_{0}}{8 \hbar}\left(\frac{c}{c_{s}}\right)^{2},
$$

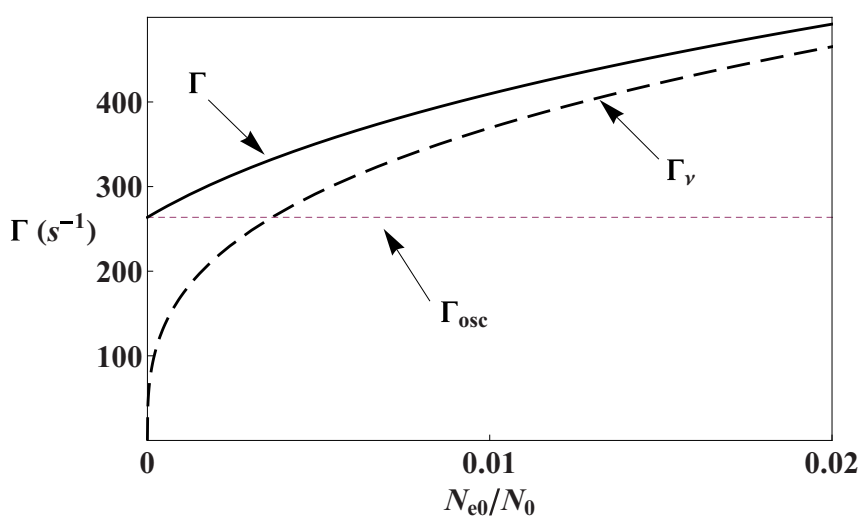

FIG. 1. Solid curve: growth rate $\Gamma$ from Eq. (19) in terms of the normalized electron-neutrino population. Dashed curve: growth rate $\Gamma_{v}$ which would take place without neutrino oscillations. Dotted horizontal line: growth rate $\Gamma_{\text {osc }}$ due uniquely to neutrino oscillations. Parameters: $n_{0}=5 \times 10^{29} \mathrm{~cm}^{-3}, N_{0}=10^{36} \mathrm{~cm}^{-3}, \mathcal{E}_{0}=1 \mathrm{MeV}$.

where $k=\Omega_{v} / c_{s}$ has been used. The unstable mode corresponds to a growth rate $\Gamma=\operatorname{Im}(\delta \omega)>0$ given by

$$
\Gamma=\left(\Gamma_{v}^{3}+\Gamma_{\text {osc }}^{3}\right)^{1 / 3},
$$

where

$$
\begin{aligned}
\Gamma_{v} & =\frac{\sqrt{3}}{2}\left(\frac{\Delta_{e}}{2}\left(\frac{c}{c_{s}}\right)^{4}\right)^{1 / 3}\left|\mathbf{k} \cdot \mathbf{v}_{0}\right|, \\
\Gamma_{\mathrm{osc}} & =\frac{\sqrt{3}}{2}\left(\frac{\Delta \Omega_{0}^{2} \mathcal{E}_{0}}{8 \hbar}\left(\frac{c}{c_{s}}\right)^{2}\right)^{1 / 3} .
\end{aligned}
$$

The quantity $\Gamma_{\nu}$ is associated with the coupling with the streaming neutrinos (with $\Omega_{v}=c_{s} k$ ). Flavor oscillations are responsible for $\Gamma_{\text {osc }}$, which can have some impact only in the case where Eq. (17) is satisfied.

The relative influence of neutrino oscillations is given by

$$
\left(\frac{\Gamma_{\text {osc }}}{\Gamma_{v}}\right)^{3}=\frac{\sqrt{2}}{64} \frac{\left(\Delta m^{2} c^{4}\right)^{2} \sin ^{2}\left(2 \theta_{0}\right)}{\left(G_{F} n_{0}\right)^{3}}\left(\frac{c_{s}}{c}\right)^{2} \frac{N_{0}}{\mathcal{E}_{0} N_{e 0}} .
$$

For an initially muonic neutrino beam $\left(N_{e 0}=0\right.$ and $N_{\mu 0}=$ $N_{0}$ ), the flavor oscillations dominate, since in this case at the beginning there are no electron-neutrinos to interact with the plasma. Lower electron densities and lower streaming neutrino energies enhance the flavor oscillation correction. Figure 1 shows the growth rates for $n_{0}=5 \times 10^{29} \mathrm{~cm}^{-3}$, $N_{0}=10^{36} \mathrm{~cm}^{-3}, \mathcal{E}_{0}=1 \mathrm{MeV}$, and varying initial electronneutrino populations, to be compared with the time scale of supernova explosions, around $1 \mathrm{~s}$ [4]. It is verified that in denser stars the neutrino oscillations play a less significant role.

The result, (22), was derived using the inverted neutrino mass hierarchy assumption

$$
\Omega\left(n_{0}\right) \approx-\Omega_{v} \approx-\sqrt{2} G_{F} n_{0} / \hbar,
$$

which is always satisfied for the dense plasma under consideration. Indeed, taking $\Delta m^{2} c^{4}=3 \times 10^{-5}(\mathrm{eV})^{2}$ and $\sin \left(2 \theta_{0}\right)=10^{-1}$ (suitable parameters solving the solar 


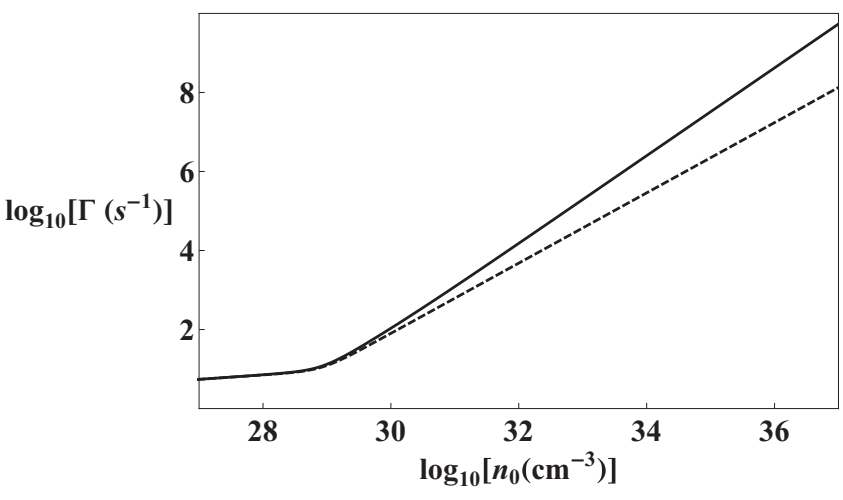

FIG. 2. Solid line: growth rate $\Gamma$ from Eq. (19) as a function of the plasma density, at logarithmic scale, for iron-core stars. Dashed line: growth rate resulting from the nonrelativistic expression of the ion-acoustic speed. Parameters: $N_{0}=N_{e 0}=10^{35} \mathrm{~cm}^{-3}, \mathcal{E}_{0}=$ $10 \mathrm{MeV}$.

neutrino deficit problem [22]), we find

$$
\frac{\sqrt{2} G_{F} n_{0}}{\hbar \omega_{0}}=2.7 \times 10^{-26} n_{0} \mathcal{E}_{0} \gg 1,
$$

where $n_{0}$ is measured in $\mathrm{cm}^{-3}$ and $\mathcal{E}_{0}$ in $\mathrm{MeV}$, which is the typical energy scale of neutrino beams in supernova progenitors [5], the inequality following from $n_{0}>10^{28} \mathrm{~cm}^{-3}$ in the standard cases [24].

The relativistic effects are depicted in Fig. 2 for the representative parameters $N_{0}=N_{e 0}=10^{35} \mathrm{~cm}^{-3}$ and $\mathcal{E}_{0}=$ $10 \mathrm{MeV}$. It is found that the growth rate increases significantly with the plasma density, which also enhances the relativistic effects present in the ion-acoustic speed. For comparison, the results from a nonrelativistic fully degenerate model are also shown, setting $\xi_{0} \equiv 0$ in Eq. (16). As is apparent, the nonrelativistic model underestimates the growth rates by a large amount, especially in denser stars. Note that in the present case the relativistic character of the plasma arises from the electron degeneracy, so that the parameter $\xi_{0}$ can have significant values.

In another potentially relevant limit, the plasma can be relativistic, due to a high thermodynamic temperature, but nondegenerate ( $T \gg T_{F}$, where $T_{F}$ is the Fermi temperature). In this situation, the equation of state would be obtained from the Jüttner distribution, and both the pressure and the number density would involve Bessel functions. Nevertheless, at the end the simple isothermal equation of state $P\left(n_{e}\right)=$ $n_{e} \kappa_{B} T$ holds in the nondegenerate case, for arbitrary relativistic strength $[25,26]$. Hence the ion-acoustic speed becomes $c_{s}^{\mathrm{ND}}=\sqrt{Z\left(d P / d n_{e}\right)_{0} / m_{i}}=\sqrt{Z \kappa_{B} T / m_{i}}$. Replacing $c_{s}$ from Eq. (16) with $c_{s}^{\mathrm{ND}}$, one has the growth rate shown in Fig. 3, where the parameters are $N_{0}=N_{e 0}=10^{35} \mathrm{~cm}^{-3}$, $\mathcal{E}_{0}=10 \mathrm{MeV}$, and $T=10 T_{F}$ (so that electrons are certainly nondegenerate). There is a qualitative similarity in comparison with the extreme degenerate case (Fig. 2). However, the nondegeneracy condition requires high temperatures, shown in Fig. 4.

Table I lists the diverse physical quantities for type II corecollapse supernova electron number densities [5,6,24,27]. For the sake of illustration, we assume an iron core so that hence-

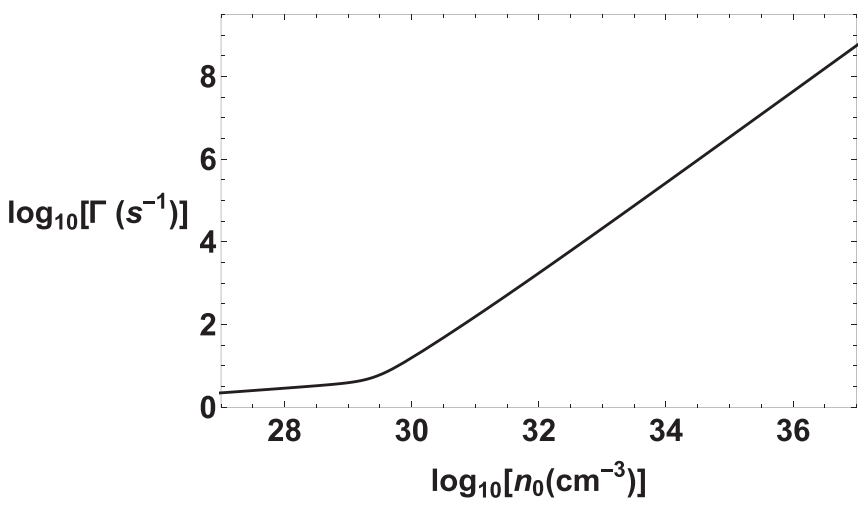

FIG. 3. Growth rate $\Gamma$ from Eq. (19) in nondegenerate plasma as a function of the plasma density, at logarithmic scale, using the nondegenerate ion-acoustic speed $c_{s}^{\mathrm{ND}}=\sqrt{Z \kappa_{B} T / m_{i}}$, for iron-core stars. Parameters: $N_{0}=N_{e 0}=10^{35} \mathrm{~cm}^{-3}, \mathcal{E}_{0}=10 \mathrm{MeV}, T=10 T_{F}$.

forth $Z=26, m_{i}=56$ a.m.u. For convenience, the Fermi temperature $T_{F}$ obtained from $\kappa_{B} T_{F}=\left(p_{F}^{2} c^{2}+m_{e}^{2} c^{4}\right)^{1 / 2}-$ $m_{e} c^{2}$ is exhibited (noting the ultradegeneracy condition $T \ll$ $\left.T_{F}\right)$. We observe that the relativistic parameter $\xi_{0}=p_{F} /\left(m_{e} c\right)$ becomes large for very dense systems. The ion-acoustic speed is always seen to be much lower than the light speed. It can also be verified that $\Omega_{v} \gg \omega_{\text {pi }}$ as required. In addition, one can define an electron coupling parameter $g=E_{p} / E_{k}$, where $E_{k} \approx \kappa_{B} T_{F}$ is a measure of the electron kinetic energy and $E_{p}=e^{2} /\left(4 \pi \epsilon_{0} r_{s}\right)$ is a measure of the electron interaction energy in terms of the Wigner-Seitz ratio $r_{s}=\left(3 / 4 \pi n_{0}\right)^{1 / 3}$. As is apparent from Table I, the electron ideality condition $g \ll 1$ is satisfied to a good approximation. Finally, besides the wave frequency $\omega \approx \Omega_{v}$, we also consider the wavelength $\lambda=2 \pi c_{s} / \Omega_{v}$, to be compared with the typical [28] iron core size $(\sim 30 \mathrm{~km})$. Using approximation $(23)$, we derive

$$
\lambda=\frac{2 \pi \hbar c_{s}}{\sqrt{2} G_{F} n_{0}},
$$

which always decreases with the density.

Another issue is the possible influence of Landau damping. Evaluating the imaginary part of the longitudinal dielectric function for ion-acoustic waves in ultradegenerate relativistic plasma, it can be proven [29] that Landau damping is not

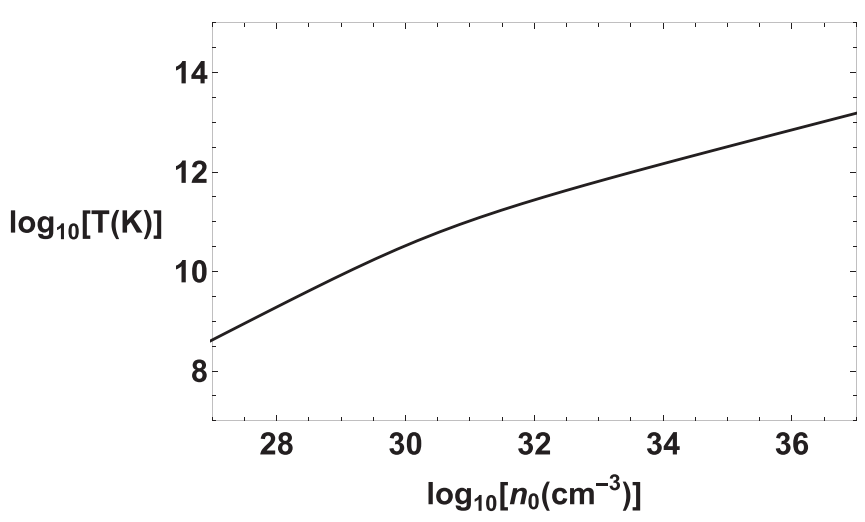

FIG. 4. Thermodynamic temperature $T=10 T_{F}$, corresponding to Fig. 3, as a function of the plasma density, at logarithmic scale. 
TABLE I. Parameters for iron-core protoneutron stars.

\begin{tabular}{lrrccl}
\hline \hline$n_{0}\left(\mathrm{~cm}^{-3}\right)$ & $T_{F}(\mathrm{MeV})$ & $p_{F} / m_{e} c$ & \multicolumn{1}{c}{$c_{s} / c$} & $g$ & \multicolumn{1}{c}{$\lambda(\mathrm{m})$} \\
\hline $10^{28}$ & 0.02 & 0.3 & $2.3 \times 10^{-3}$ & $3.0 \times 10^{-2}$ & $7.2 \times 10^{3}$ \\
$10^{30}$ & 0.29 & 1.2 & $8.8 \times 10^{-3}$ & $8.1 \times 10^{-3}$ & 0.6 \\
$10^{32}$ & 2.37 & 5.5 & $2.2 \times 10^{-2}$ & $4.5 \times 10^{-3}$ & 0.1 \\
$10^{34}$ & 12.68 & 25.7 & $4.7 \times 10^{-2}$ & $3.9 \times 10^{-3}$ & 0.03 \\
$10^{36}$ & 60.70 & 119.5 & $1.0 \times 10^{-1}$ & $3.8 \times 10^{-3}$ & 0.008 \\
\hline \hline
\end{tabular}

relevant as long as $p_{F} /\left(m_{i} c\right) \ll 1$, which is satisfied except if $n_{0} \gg 10^{44} \mathrm{~cm}^{-3}$ (neutron star densities).

\section{CONCLUSION}

In this work, we reformulate and generalize the treatment of [8], now taking into account the degenerate and relativistic conditions of protoneutron stars originating type II corecollapse supernovas. The main results are the development of a general model for neutrino-plasma interactions taking into account neutrino flavor oscillations, in degenerate relativistic plasmas. Unstable wavelengths and resonance conditions with neutrino oscillations are found, destabilizing ionacoustic waves in extreme relativistic astrophysical scenarios. In comparison with the free energy source from the streaming neutrinos, the neutrino oscillations play a more significant role in the ion-acoustic wave destabilization, only if the initial nonelectronic neutrino population dominates. No quantum diffraction (Bohm potential) was included because it can be estimated [8] as an exceedingly small contribution for the wavelengths where the double-resonance condition, (17), is met. Likewise, exchange-correlation potentials [30,31] have not yet been considered since, to our knowledge, currently there are no known relativistic quantum hydrodynamical equations with exchange correlation taken into account. Future developments could involve finite electron temperatures, finite neutrino beam temperatures, and collisional effects. Finally, we expect that the instabilities should enhance the coupling of the neutrino beam with the plasma, especially taking into account the high-amplitude waves developing from the unstable linear waves. It would be very interesting to compare the resulting coupling with those currently considered in corecollapse supernova models.

\section{ACKNOWLEDGMENTS}

The author acknowledges financial support from $\mathrm{CNPq}$ (Conselho Nacional de Desenvolvimento Científico e Tecnológico), Brazil.
[1] A. Taroni, Nature Phys. 11, 891 (2015).

[2] R. Bingham, J. M. Dawson, J. J. Su, and H. A. Bethe, Phys. Lett. A 193, 279 (1994); J. T. Mendonça, R. Bingham, P. K. Shukla, J. M. Dawson, and V. N. Tsytovich, ibid. 209, 78 (1995).

[3] L. O. Silva, R. Bingham, J. M. Dawson, J. T. Mendonça, and P. K. Shukla, Phys. Rev. Lett. 83, 2703 (1999).

[4] L. O. Silva and R. Bingham, J. Cosmol. Astropart. Phys. (2006) 011.

[5] K. Hirata, T. Kajita, M. Koshiba, M. Nakahata, Y. Oyama, N. Sato, A. Suzuki, M. Takita, Y. Totsuka, T. Kifune et al., Phys. Rev. Lett. 58, 1490 (1987).

[6] J. M. Laming, New Astron. 4, 389 (1999).

[7] T. Tajima and K. Shibata, Plasma Astrophysics (AddisonWesley, Reading, MA, 1997).

[8] F. Haas, K. A. Pascoal, and J. T. Mendonça, Phys. Rev. E 95, 013207 (2017).

[9] F. Haas, in 9th International Conference on the Frontiers of Plasma Physics and Technology (FPPT-9), Colombo, Sri Lanka, 8-12 April 2019 (unpublished), https://www.fpptseries.org.

[10] S. Weinberg, Gravitation and Cosmology (John Wiley and Sons, New York, 1972).

[11] J. T. Mendonça and F. Haas, Phys. Plasmas 20, 072107 (2013).

[12] F. Haas, K. A. Pascoal, and J. T. Mendonça, Phys. Plasmas 24, 052115 (2017).

[13] F. Haas, K. A. Pascoal, and J. T. Mendonça, Phys. Plasmas 23, 012104 (2016); R. J. Fitzgerald, Phys. Today 69(3), 20 (2016).

[14] F. Haas and K. A. Pascoal, Phys. Plasmas 24, 092109 (2017).
[15] F. Haas, K. A. Pascoal, and J. T. Mendonça, Phys. Rev. D 96, 023018 (2017).

[16] S. Chandrasekhar, Mon. Not. R. Astron. Soc. 95, 207 (1935).

[17] F. Haas, J. Plasma Phys. 82, 705820602 (2016).

[18] L. O. Silva, R. Bingham, J. M. Dawson, J. T. Mendonça, and P. K. Shukla, Phys. Plasmas 7, 2166 (2000).

[19] A. J. Brizard, H. Murayama, and J. S. Wurtele, Phys. Rev. E 61, 4410 (2000).

[20] A. Serbeto, Phys. Lett. A 296, 217 (2002).

[21] F. Suekane, Neutrino Oscillations-A Practical Guide to Basics and Applications (Springer, Tokyo, 2015).

[22] G. G. Raffelt, Stars as Laboratories for Fundamental Physics (University of Chicago Press, Chicago, 1996).

[23] A. Serbeto, P. K. Shukla, and L. F. Monteiro, Plasma Phys. Control. Fusion 44, L43 (2002).

[24] N. Yamamoto, Phys. Rev. D 93, 065017 (2016).

[25] A. Weiss, W. Hillebrandt, H. C. Thomas, and H. Ritter, Cox and Giuli's Principles of Stellar Structure, Advances in Astronomy and Astrophysics (Cambridge Scientific, Cambridge, UK, 2006).

[26] G. Faussurier, Phys. Plasmas 23, 122704 (2016).

[27] T. Yoshida, K. Takahashi, and H. Umeda, J. Phys.: Conf. Ser. 718, 062073 (2016).

[28] S. E. Wooley and H.-T. Janka, Nat. Phys. 1, 147 (2005).

[29] A. E. Delsante and N. E. Frankel, Ann. Phys. 125, 135 (1980).

[30] N. Crouseilles, P.-A. Hervieux, and G. Manfredi, Phys. Rev. B 78, 155412 (2008).

[31] F. Haas, Plasma Phys. Control. Fusion 61, 044001 (2019). 